\title{
Two-Degree-of-Freedom Control of a Dual-Stage Actuator Positioning System for Short-Span Tracking
}

\author{
Jinchuan Zheng, Weizhou Su, and Minyue Fu
}

\begin{abstract}
This paper presents a new two-degree-of-freedom (2DOF) control design method for a dual-stage actuator (DSA) positioning system consisting of a linear motor (LM) and a piezo actuator (PA). The $2 \mathrm{DOF}$ controller is proposed to achieve disturbance rejection and short-span tracking in the PA range and is designed using the doubly coprime factorization (DCF) approach, with which the closed-loop transfer function is expressed explicitly in terms of design parameters. This greatly simplifies the optimization of design parameters in meeting desired specifications. We further study how to use the design parameters to deal with specific problems in the DSA, i.e., control allocation and trajectory planning. Experimental results demonstrate the practical implementation of the DSA control system and verify its effectiveness for step tracking and disturbance rejection.
\end{abstract}

\section{INTRODUCTION}

Dual-stage actuator (DSA) servo systems typically consist of a coarse actuator and a fine actuator connected in series to provide long travel range, high positioning accuracy and fast response. The industrial applications include the dualstage hard disk drive (HDD) actuator [1], the dual-stage XY positioning tables [2], and so on. The control design for the DSA is a challenging task because 1) The DSA system is a dual-input single-output (DISO) system, which means that for a given desired trajectory, inputs to the two actuators are not unique. Thus, a proper control strategy is required for control allocation. 2) The secondary actuator typically has a very limited travel range, which results in a severe actuator saturation problem. A number of approaches have been reported to deal with dual-stage control problems. Control design for reference following can be found in [3], [4]. In [5] and [6], the secondary actuator saturation problem is explicitly taken into account during the control design. In [7], a decoupled track-seeking controller is developed to enable high-speed short-span seeking for a dual-stage HDD servo system. Further, short and long-span seeking controls are incorporated in a single control scheme with fast settling time [8], [9].

In this paper, we present a new control design method for a DSA positioning system consisting of a linear motor (LM) and a piezo actuator (PA). We focus on the development of a two-degree-of-freedom (2DOF) controller for disturbance

J. Zheng and M. Fu are with the School of Electrical Engineering and Computer Science, The University of Newcastle, Callaghan, NSW 2308, Australia. Jinchuan.Zheng@newcastle.edu.au ; Minyue.Fu@newcastle.edu.au

W. Su is with the College of Automation Science and Engineering, South China University of Technology, Guangzhou, China.

This work was supported in part by a Research Grant from The University of Newcastle, Australia, and in part by the Natural Science Foundation of China under the grants $60834003,60774057$. rejection and step tracking in the PA range. A doubly coprime factorization (DCF) [10] is used for the 2DOF controller design because it can provide the advantages that: 1) it parameterizes all linear internally stabilized 2DOF controller by two free design parameters; 2) it offers a unifying design method to solve the tracking and disturbance rejection problems; 3 ) the derived frequency transfer functions of disturbance rejection response and seeking response are simply expressed and they are uniquely in terms of the design parameters, which makes the relationship between the design parameters and the desired specifications explicit. Compared with the existing DSA control methods, the proposed 2DOF controller explicitly addresses both the step tracking and disturbance rejection problem in a unifying design framework and gives the solution to a specified performance index indicating the tracking performance. Further, this paper has first revealed that the tracking performance of asymptotical tracking and disturbance rejection is equivalent to two independent optimization problems. This result obviously can decouple the design of the controller and thus simplify the design process. Finally, we verify the effectiveness of the DSA controller through experiment results. For tracking control beyond the $\mathrm{PA}$ range, it is not the purpose of this paper and thereby is not given in detail. Interested reads can refer to [9] for the control strategy in this range.

Throughout this paper, we use the following notation. For any signal $u(t)$, we denote its Laplace transform by $\hat{u}(s)$. $\|\cdot\|$ denotes the Euclidean vector norm and $\|\cdot\|_{2}$ the norm in space $L_{2}$.

\section{2DOF CONTROL DESIGN BASED ON DCF}

The 2DOF control systems are the most general feedback configuration in linear control schemes. Fig. 1 shows a generic structure for this class of systems. In this setup, $G$ denotes the given linear time-invariant (LTI) plant model, $W$ denotes the known LTI stable and proper weight, and $K$ denotes the 2DOF controller to be designed. The signals $r$, $y, u$, and $d$ represent, respectively, the step reference signal, the system output, the control input, and the disturbance with energy bounded by $\delta^{2}$, i.e., $\|d\|_{2}^{2} \leq \delta^{2}$.

In this paper, we consider the asymptotic tracking and disturbance rejection problem for the system in Fig. 1. We need to design the controller $K$ such that the closedloop system is internally stable and the system output $y$ asymptotically tracks a step signal $r(t)=v, t \geq 0$ for all disturbance $d \in L_{2}$ with $\|d\|_{2} \leq \delta$. The measure of the 


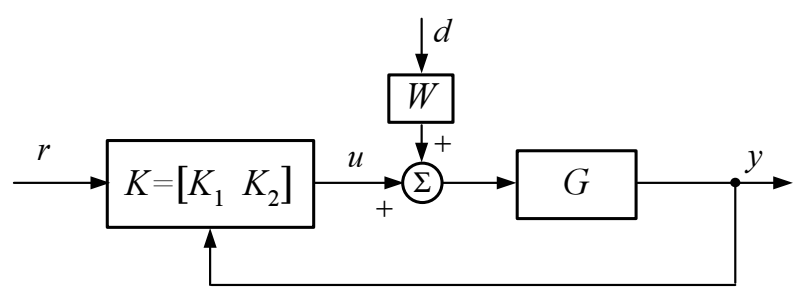

Fig. 1. A generic 2DOF control system.

tracking performance is defined as

$$
J=\int_{0}^{\infty}\|e(t)\|^{2} d t,
$$

where $e(t)=r(t)-y(t)$ denotes the tracking error. Obviously, $J$ depends on the disturbance $d$. We thus consider the worst value of $J$ over all possible $d$ as the performance index for the tracking and disturbance rejection problem, i.e.,

$$
\sup _{\|d\|_{2} \leq \delta} J
$$

Therefore, it is our interest to seek a controller $K$ among all possible stabilizing 2DOF controllers to achieve the minimum value of (2) defined by

$$
J_{o p t}=\inf _{K} \sup _{\|d\|_{2} \leq \delta} J .
$$

The DCF is a well-suited approach to solve (3). Let $\mathcal{R H}_{\infty}$ denotes the set of all stable, proper, rational transfer function matrices. Let also the right and left coprime factorizations of $G$ be given by

$$
G=N D^{-1}=\tilde{D}^{-1} \tilde{N}
$$

where $N, D, \tilde{N}, \tilde{D} \in \mathcal{R} \mathcal{H}_{\infty}$ and satisfy the doubly Bezout identify

$$
\left[\begin{array}{cc}
\tilde{X} & -\tilde{Y} \\
-\tilde{N} & \tilde{D}
\end{array}\right]\left[\begin{array}{ll}
D & Y \\
N & X
\end{array}\right]=I
$$

for some $X, Y, \tilde{X}, \tilde{Y} \in \mathcal{R H}_{\infty}$.

In [11], Nett has proposed explicit formulas for the doubly coprime fractional representation of an LTI system in terms of its state-space realization. This method is numerically easy to use. To do this, we first represent the plant model $G(s)$ in state-space as follows:

$$
G(s)=C(s I-A)^{-1} B,
$$

where $A, B$, and $C$ are matrices with appropriate dimensions. Suppose the pairs $(A, B)$ and $(A, C)$ are stabilizable and detectable, respectively. Select $F$ and $L$ such that $(A-B F)$ and $(A-L C)$ are both Hurwitz. Thus, a DCF of $G$ is given by

$$
\left\{\begin{array}{l}
N(s)=C(s I-A+B F)^{-1} B \\
D(s)=I-F(s I-A+B F)^{-1} B \\
\tilde{N}(s)=C(s I-A+L C)^{-1} B \\
\tilde{D}(s)=I-C(s I-A+L C)^{-1} L \\
X(s)=I+C(s I-A+B F)^{-1} L \\
\tilde{X}(s)=I+F(s I-A+L C)^{-1} B \\
Y(s)=-F(s I-A+B F)^{-1} L \\
\tilde{Y}(s)=-F(s I-A+L C)^{-1} L .
\end{array}\right.
$$

According to [10], all linear internally stabilizing 2DOF controllers $K=\left[\begin{array}{ll}K_{1} & K_{2}\end{array}\right]$ can be parameterized by

$$
\begin{aligned}
\hat{u} & =K_{1} \hat{r}+K_{2} \hat{y}, \\
K_{1} & =(\tilde{X}-R \tilde{N})^{-1} Q, \\
K_{2} & =(\tilde{X}-R \tilde{N})^{-1}(\tilde{Y}-R \tilde{D}),
\end{aligned}
$$

where $Q, R \in \mathcal{R} \mathcal{H}_{\infty}$ are the free parameters to be designed. By substituting the controllers $K_{1}, K_{2}$ and the factorized plant model (4) into Fig. 1, we can easily obtain the following input-output relationship in frequency domain

$$
\begin{aligned}
\hat{y} & =T_{y r} \hat{r}+T_{y d} \hat{d}, \\
T_{y r} & =N Q, \\
T_{y d} & =(X-N R) \tilde{N} W,
\end{aligned}
$$

where $T_{y r}$ and $T_{y d}$ denote the closed-loop responses from the reference and disturbance to the system output, respectively. It is advantageous that the closed-loop response functions are expressed by the design parameters $Q$ and $R$ explicitly. Hence, from Parseval's theorem, we have

$$
\begin{aligned}
J & =\int_{0}^{\infty}\|e(t)\|^{2} d t=\|\hat{r}-\hat{y}\|_{2}^{2} \\
& =\left\|\left(I-T_{y r}\right) \hat{r}-T_{y d} \hat{d}\right\|_{2}^{2} .
\end{aligned}
$$

Then, the following result is clear.

Theorem 1 [12]: Let $G$ have non-minimum phase (NMP) zeros $z_{1}, z_{2}, \cdots, z_{m}$ with corresponding Blaschke vectors $\eta_{1}, \eta_{2}, \cdots, \eta_{m}$. Then the minimax tracking performance of asymptotical tracking and disturbance rejection of the system is given by

$$
\begin{aligned}
J_{\text {opt }} & =\inf _{Q}\left\|\left(I-T_{y r}\right) \hat{r}\right\|_{2}^{2}+\delta^{2} \inf _{R}\left\|T_{y d}\right\|_{\infty}^{2} \\
& =2 \sum_{i=1}^{m} \frac{\operatorname{Re}\left(z_{i}\right)}{\left|z_{i}\right|^{2}} \cos ^{2} \angle\left(\eta_{i}, v\right)+\delta^{2} \inf _{R}\left\|T_{y d}\right\|_{\infty}^{2}(14)
\end{aligned}
$$

Remark 1: The theorem reveals that the optimal tracking performance with the 2DOF controller is a sum of two terms as shown in (13). The first term is the optimal tracking performance of the system without the disturbance input $d$, while the second one is the best achievable performance of disturbance attenuation of the system without the reference signal $r$. These two optimal problems have been studied in [13], [14], respectively, the results therein are then applied to yield (14).

From the controller design point of view, Theorem 1 also implies that to achieve $J_{\text {opt }}$ is equivalent to two independent optimization problems in terms of the free parameters $R$ and $Q$, respectively. More specific, these two problems are

1) Design $R \in \mathcal{R} \mathcal{H}_{\infty}$ to minimize $\|(X-N R) \tilde{N} W\|_{\infty}$,

2) Design $Q \in \mathcal{R} \mathcal{H}_{\infty}$ to minimize $\|(I-N Q) \hat{r}\|_{2}^{2}$. Intuitively, one may simply choose $R=N^{-1} X$ and $Q=$ $N^{-1}$ to yield $J_{o p t}=0$. However, this option only applies to the special case where the plant must be proper, right invertible, stable and minimum phase, the resulting $R$ and $Q$ are proper and the control input has no saturation. In practical servomechanisms, these strict conditions are rarely satisfied 


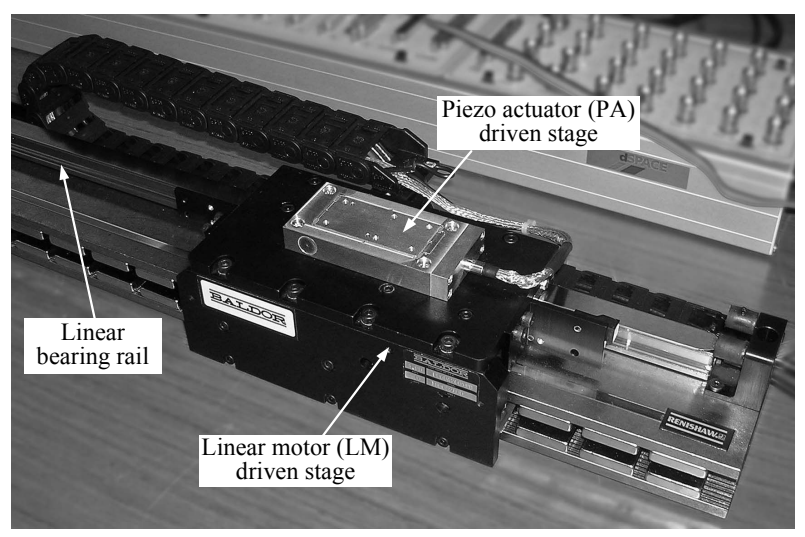

Fig. 2. A DSA positioning system.

at the same time. Instead, the designer has to deal with one or more of the constraints. In addition, the optimal $Q$ would be related to the reference signal of the system. Therefore, the design of $R$ and $Q$ requires some extra techniques to obtain a practical servo system without degrading the tracking performance significantly. In general, we can attempt to design $R$ and $Q$ such that $T_{y d}=(X-N R) \tilde{N} W \rightarrow 0$ and $T_{y r}=N Q \rightarrow I$ in the frequency of interest according to the design specifications [15]. Under this circumstance, a suboptimal controller is achieved to approximate the optimal one that yields (14), while to handle the constraints at hand. The performance of the resulting servo system will then compromise among the optimal tracking, robustness, and easy implementation (e.g., least controller order). The design examples along this line include [16] and [17] that tackle NMP flexible beams and actuator torque constrains.

In the next section, we will apply such a 2DOF controller design approach to a DSA servo system and discuss how to select $R$ and $Q$ to achieve both fast step tracking and disturbance rejection.

\section{Application to a DSA CONTROL System}

\section{A. Plant Modeling}

The DSA positioning system is depicted in Fig. 2, which consists of a primary stage driven by an LM and a secondary stage driven by a PA. The LM has a $0.5 \mathrm{~m}$ travel range, while the PA has a limited travel range of $\pm 15 \mu \mathrm{m}$. The nonlinear friction force of the LM is overcome by a precompensator, see [9] for details. The resonance of the PA stage flexure is actively damped by its integrated control electronics. In this setup, we can simply ignore the coupling forces between the two actuators [9]. After these manipulations, the LM model is approximated by:

$$
G_{1}=\frac{\hat{y}_{1}}{\hat{u}_{1}}=\frac{k_{1}}{s^{2}},
$$

where $y_{1}$ is the LM position output, $u_{1}$ is the control input with $\left|u_{1}\right| \leq \bar{u}_{1}=1 \mathrm{~V}$, and $k_{1}=1.5 \times 10^{7}$. The PA model is approximated by:

$$
G_{2}=\frac{\hat{y}_{2}}{\hat{u}_{2}}=\frac{k_{2}}{s^{2}+a s+b},
$$
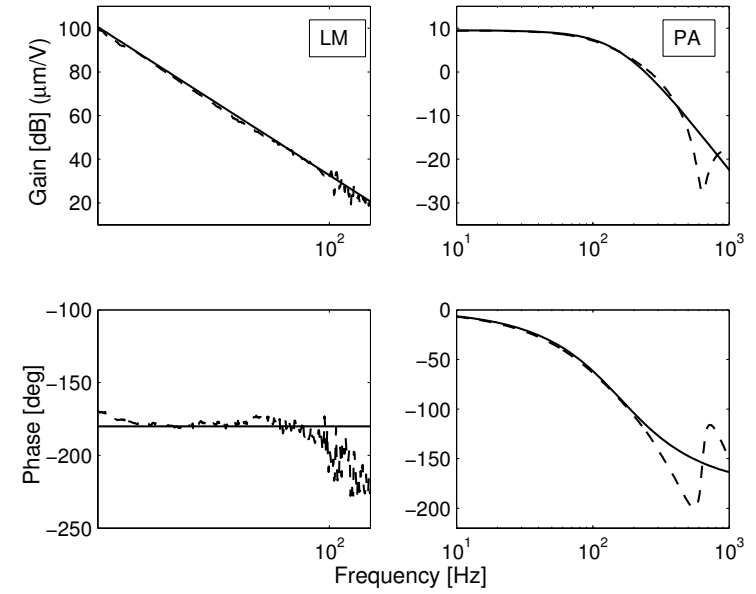

Fig. 3. Frequency responses of the DSA plant model (Solid lines: Identified, Dashed lines: Measured).

where $y_{2}$ is the PA position output relative to the LM, $u_{2}$ is the control input with $\left|u_{2}\right| \leq \bar{u}_{2}=5 \mathrm{~V}$, and $k_{2}=3.0 \times 10^{6}$, $a=1810, b=1.0 \times 10^{6}$. Fig. 3 shows the frequency responses of the LM and PA system, which verify the accuracy of the identified models in the frequency of interest.

The control output $y=y_{1}+y_{2}$, i.e., the absolute position of the PA, is the only available measured output for feedback control. Hence, the overall DSA model $G$ can be represented as a DISO linear system

$$
\hat{y}=G \hat{u}=\left[\begin{array}{ll}
G_{1} & G_{2}
\end{array}\right]\left[\begin{array}{l}
\hat{u}_{1} \\
\hat{u}_{2}
\end{array}\right] .
$$

To get the DCF of $G$ by (7), we then transform $G$ into a state-space form in terms of (6). For the disturbance source, we are concerned with a shock disturbance acting on the LM. The standard half sine wave with a duration of $10 \mathrm{~ms}$ is typically used as the shock test [18]. Thus, we can model the disturbance as

$W=\left[\begin{array}{c}\frac{0.05}{0.0008 s+1} \\ 0\end{array}\right], d=\left\{\begin{array}{cl}\sin (314 t), & t \in\left[\begin{array}{ll}0 & 0.01\end{array}\right] \\ 0, & \text { otherwise. }\end{array}\right.$

Obviously, we have $\|d\|_{2} \leq \delta=0.071$.

\section{B. 2DOF Controller Design}

The 2DOF controller for the DSA step responses within the PA Range should satisfy the following specifications:

1) The overshoot should be kept under $1 \mu \mathrm{m}$.

2) The control inputs to the LM and PA are not saturated, i.e., should not exceed \pm 1 and $\pm 5 \mathrm{~V}$, respectively.

3) In response to a step reference, the displacement of PA should settle down to zero at steady state such that it can further response to a sequential reference.

4) The DSA servo system should have gain margin larger than $6 \mathrm{~dB}$ and phase margin more than $50 \mathrm{deg}$.

We will present a step-by-step design procedure.

Step 1: DCF of $G$

According to (7), we should first select $F$ and $L$ such that $(A-B F)$ and $(A-L C)$ are both Hurwitz. Clearly, $F$ is a state feedback gain matrix, and $L$ is a state estimator gain 


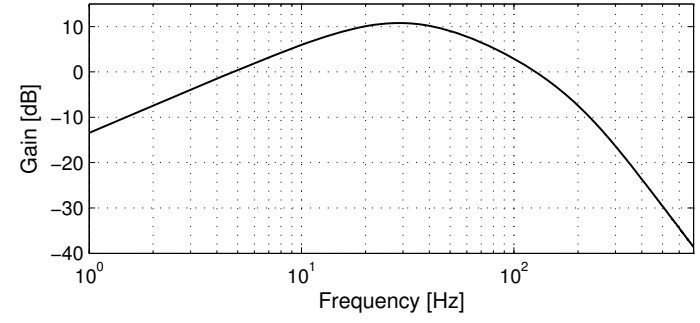

Fig. 4. Bode plot of disturbance rejection response $T_{y d}$.

matrix. Since there is no coupling between the LM and the PA, the gains $F$ and $L$ can be partitioned as

$$
F=\left[\begin{array}{c|c}
F_{1} & 0 \\
\hline 0 & F_{2}
\end{array}\right], \quad L=\left[\begin{array}{c}
L_{1} \\
\hline L_{2}
\end{array}\right] .
$$

Hence, we can individually design the gains for the LM and PA loops by using the pole placement method such that the PA loop should have a faster dynamics than the LM loop, and the estimator is faster than the state feedback loop. To do this, we select $F_{1}=\left[\begin{array}{ll}0.0024 & 2.2 \times 10^{-5}\end{array}\right]$ and $L_{1}=$ $\left[30373.8 \times 10^{6}\right]^{T}$ to make the LM loop and its estimator have a bandwidth of 30 and $200 \mathrm{~Hz}$, respectively, and select $F_{2}=-\left[\begin{array}{lll}0.286 & 3.7 \times 10^{-4}\end{array}\right]$ and $L_{2}=\left[\begin{array}{lll}243 & -3.6 \times 10^{5}\end{array}\right]^{T}$ for the counterparts of the PA with 60 and $250 \mathrm{~Hz}$ bandwidths, respectively. Then, the DCF of $G$ can be computed by (7). Step 2: Design of $R$

For disturbance rejection, we should make the disturbance rejection function $T_{y d}=(X-N R) \tilde{N} W \rightarrow 0$ in the low frequencies. Let $R=\left[\begin{array}{ll}R_{1} & R_{2}\end{array}\right]^{T}$ and $N=\left[\begin{array}{ll}N_{1} & N_{2}\end{array}\right]$, we then take

$$
R_{1}=N_{1}^{-1} X r_{1}(s), \quad R_{2}=N_{2}^{-1} X r_{2}(s),
$$

with

$$
r_{1}(s)=\frac{1-\beta}{(\eta s+1)^{2}}, \quad r_{2}(s)=\frac{\beta}{(\eta s+1)^{2}},
$$

where $\eta>0$ and $\beta \in\left[\begin{array}{ll}0 & 1\end{array}\right]$ are tuning scalars. Note that the order of $r_{1,2}$ is chosen to make $R_{1,2}$ proper at least. Then, we have

$$
T_{y d}=\left(1-\frac{1}{(\eta s+1)^{2}}\right) X \tilde{N} W .
$$

We can see that the term $\left(1-1 /(\eta s+1)^{2}\right)$ introduces low gains in low frequencies for disturbance rejection. Moreover, the available frequency region for the disturbance rejection problem can be increased with a smaller $\eta$. Since stability margin is also required for robustness, we can study the openloop characteristics of the DSA, which is defined by

$$
O L(s)=G K_{2}=\frac{Y_{1} G_{1}+Y_{2} G_{2}-X /(\eta s+1)^{2}}{X\left(1-1 /(\eta s+1)^{2}\right)},
$$

where $Y_{1}$ and $Y_{2}$ are, respectively, the elements of $Y$ with $Y=\left[\begin{array}{ll}Y_{1} & Y_{2}\end{array}\right]^{T}$. It is clear that the open-loop transfer function is related to $\eta$ only. However, the relationship between the stability margin and $\eta$ is implicit. Hence, we have to tune $\eta$ by trial and error such that a suitable stability
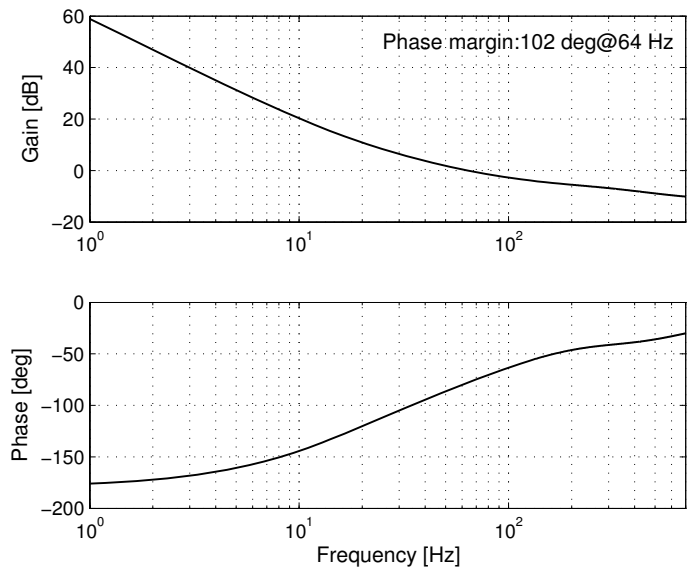

Fig. 5. Bode plot of $\Gamma$ function indicating DSA control allocation.

margin and disturbance rejection function in (21) are both achieved.

Next, we discuss how to select $\beta$. In fact, for a given $T_{y d}$ (or equivalently a given position output), $\beta$ is related to the allocation of the control efforts of the two actuators. Typically, the LM works mainly for the low-frequency movement, while the PA responses more for high-frequency disturbance. With such allocation in frequency domain, it is possible to take full advantage of the PA to bypass the LM uncertainty in the high-frequency band and improve the servo bandwidth. A key point to analyze the control allocation of the two actuators is the intersection of the two paths in frequency domain. Specifically, we can analyze the ratio of the open-loop systems of the two actuators. This idea is identical to the so-called $P Q$ method [3]. Let the controller $K_{2}=\left[\begin{array}{ll}K_{21} & K_{22}\end{array}\right]^{T}$, and then define the open-loop systems of the LM and PA as $O L_{1}=G_{1} K_{21}$ and $O L_{2}=G_{2} K_{22}$, respectively. Then we can obtain the ratio of $O L_{1}$ and $O L_{2}$ as

$$
\Gamma=\frac{O L_{1}}{O L_{2}}=\frac{X(1-\beta)-(\eta s+1)^{2} Y_{1} G_{1}}{X \beta-(\eta s+1)^{2} Y_{2} G_{2}} .
$$

We can see that $\Gamma$ is a function of $\beta$ provided that $\eta$ is determined. In order to make the two actuators have maximum cooperation, $\Gamma$ is chosen to give a roll-off characteristics and a phase margin of at least $60 \mathrm{deg}$ at the $0-\mathrm{dB}$ crossover frequency [3].

In our case, we choose $\eta=1 /(2 \pi 250)$ and $\beta=0.8$. The corresponding Bode plot of the $T_{y d}$ is shown in Fig. 4. Fig. 5 shows the phase margin of 102 degree for $\Gamma$ function as desired. To check the stability margin, Fig. 6 shows the openloop system of the DSA, which achieves a phase margin of $54 \mathrm{deg}$ at $146 \mathrm{~Hz}$, and a gain margin of $\infty$. Compared with the LM loop whose phase margin is only $13 \mathrm{deg}$ at $84 \mathrm{~Hz}$, we can see that the PA loop improves the stability margin and pushes the open-loop frequency bandwidth.

Step 3: Design of $Q$

Let $Q=\left[\begin{array}{ll}Q_{1} & Q_{2}\end{array}\right]^{T}$, Due to the fact that $G_{1}$ and $G_{2}$ are minimum phase, we thus aim at the design of $Q_{1}$ and $Q_{2}$ such that $T_{y r}=N_{1} Q_{1}+N_{2} Q_{2} \rightarrow 1$ has a high frequency 

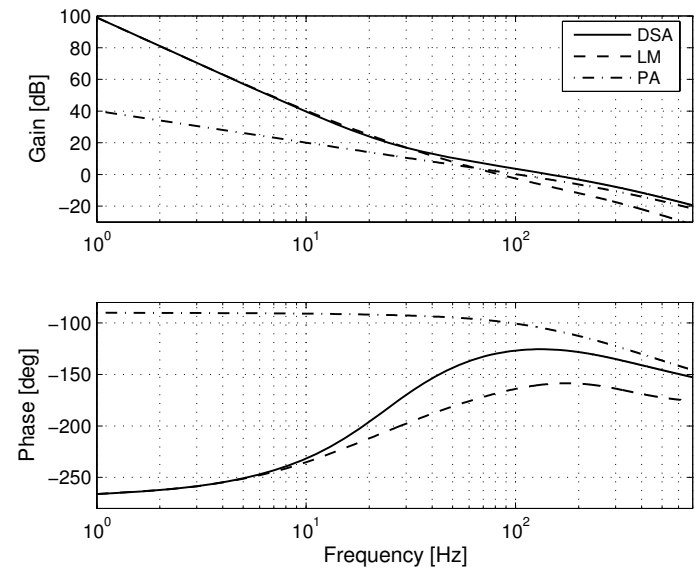

Fig. 6. Bode plot of open-loop systems.

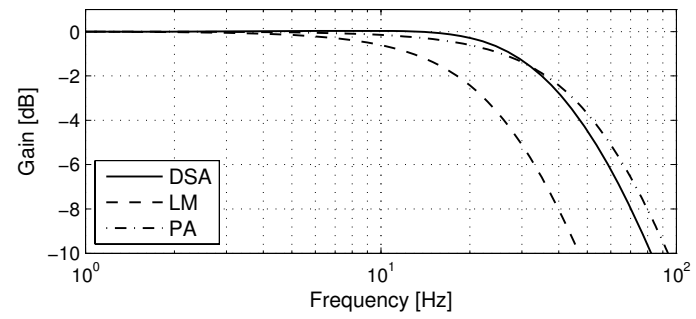

Fig. 7. Bode plot of the DSA closed-loop systems.

bandwidth and the control inputs for a step response are within both actuators' control limits. Furthermore, it is required that the displacement of PA settles down to zero at steady state. This means that $y_{1}(\infty)=r$ and $y_{2}(\infty)=0$ should be satisfied for a step response with amplitude $r$ and the disturbance with $d(\infty)=0$. Hence, we first analyze the individual outputs of the two actuators. Partition $D$ as

$$
D=\left[\begin{array}{cc}
D_{1} & 0 \\
0 & D_{2}
\end{array}\right],
$$

and suppose $d=0$, it is thus easy to get

$$
\begin{aligned}
{\left[\begin{array}{l}
\hat{y}_{1} \\
\hat{y}_{2}
\end{array}\right] } & =\left[\begin{array}{cc}
G_{1} & 0 \\
0 & G_{2}
\end{array}\right]\left[\begin{array}{l}
\hat{u}_{1} \\
\hat{u}_{2}
\end{array}\right] \\
& =\left[\begin{array}{cc}
N_{1} D_{1}^{-1} & 0 \\
0 & N_{2} D_{2}^{-1}
\end{array}\right] D Q \hat{r} \\
& =\left[\begin{array}{c}
N_{1} Q_{1} \\
N_{2} Q_{2}
\end{array}\right] \hat{r} .
\end{aligned}
$$

We can see that the step responses of the two actuators are completely decoupled in terms of $Q_{1}$ and $Q_{2}$. As the transfer functions $N_{1}$ and $N_{2}$ have been properly designed in Step 1 to individually reflect the LM and PA closed-loop dynamics, we can then interpret $Q_{1}$ and $Q_{2}$ as the trajectory planning functions for the two actuators.

From Theorem 1, we can infer that the minimal $\|(I-$ $N Q) \hat{r} \|_{2}^{2}$ achievable is zero as the DSA model has no NMP zeros. This can be completed by selecting $Q_{1}=N_{1}^{-1}$ and $Q_{2}=0$, which, however, is not a practical solution due to the improper $Q_{1}$ and the saturation of $u_{1}$. In order to

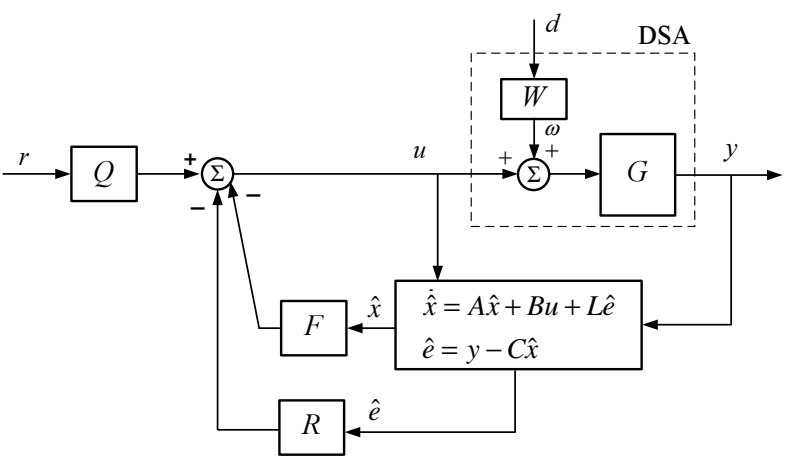

Fig. 8. DSA controller structure for practical implementation.

compromise between the tracking speed and the limitation of the control input, we choose $Q_{1}$ and $Q_{2}$ as

$$
\begin{aligned}
& Q_{1}=N_{1}(0)^{-1}, \\
& Q_{2}=\gamma N_{2}(0)^{-1}\left(1-N_{1} N_{1}(0)^{-1}\right),
\end{aligned}
$$

where $\gamma \in\left[\begin{array}{ll}0 & 1\end{array}\right]$ is a tuning parameter. It is obvious that $N_{1}(0) Q_{1}(0)=1$ and $N_{2}(0) Q_{2}(0)=0$, which imply that

$$
y(\infty)=y_{1}(\infty)+y_{2}(\infty)=r+0=r .
$$

Moreover, define the LM and PA closed-loop dynamics by

$$
T_{1}=N_{1} N_{1}(0)^{-1}, T_{2}=N_{2} N_{2}(0)^{-1} .
$$

We then have the step response transfer function of the DSA

$$
T_{y r}=T_{1}+\gamma T_{2}\left(1-T_{1}\right)
$$

It is clear that when $\gamma$ varies from 0 to 1 , the cut-off frequency of $T_{y r}$ switches from that of $T_{1}$ to that of $T_{2}$. On the other hand, we can see from (27) that the PA will follow the scaled tracking error of the LM loop $\left(\gamma\left(1-N_{1} N_{1}(0)^{-1}\right) r\right)$, where $\gamma$ actually determines the contribution of the PA to the overall position output. Since the PA has a faster response than the LM loop, it is preferable to have a maximal position output of the PA. Thus, we should maximize $\gamma \in\left[\begin{array}{ll}0 & 1\end{array}\right]$ subject to $\left\|T_{y r}\right\|_{\infty} \leq 1.067$ and $\left\|u_{2}\right\|_{\infty} \leq 5 \mathrm{~V}$, which are introduced for an overshoot under $1 \mu \mathrm{m}$ and for no saturation of the PA, respectively. For the LM, its control input $u_{1}$ is generally not saturated for step responses within the PA range. Otherwise, we have to go back to Step 1 and reduce $F_{1}$ for slower LM dynamics. Although this iteration can be avoided by adding extra tunable dynamics to (26) to generate a slower trajectory for the LM, we believe it is not costeffective as the selection of $Q_{1}$ as a constant gain can reduce the overall controller order. In our case, we obtain $\gamma=0.5$ to meet the requirement. Fig. 7 shows the Bode plot of the closed-loop systems for the $\operatorname{DSA}\left(T_{y r}\right)$, the $\operatorname{LM}\left(T_{1}\right)$, and the $\mathrm{PA}\left(T_{2}\right)$, respectively.

\section{EXPERIMENTAL RESULTS}

Experiments are conducted to verify the effectiveness of the proposed DSA controller. Fig. 8 shows an equivalent 2DOF controller structure for practical implementation. Compared the lumped 2DOF controller computed by (9) and 


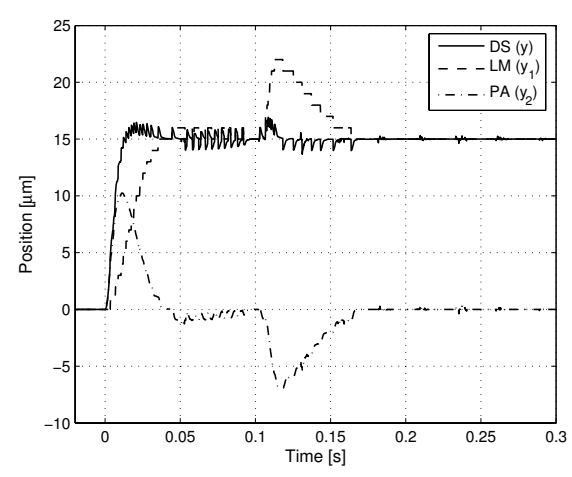

(a) Dual-stage actuator

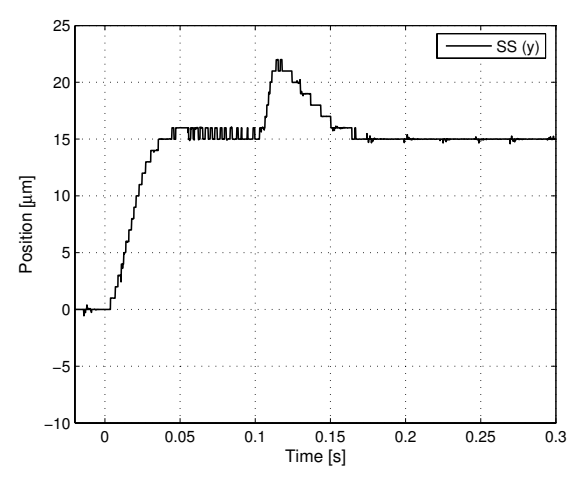

(b) Single-stage actuator

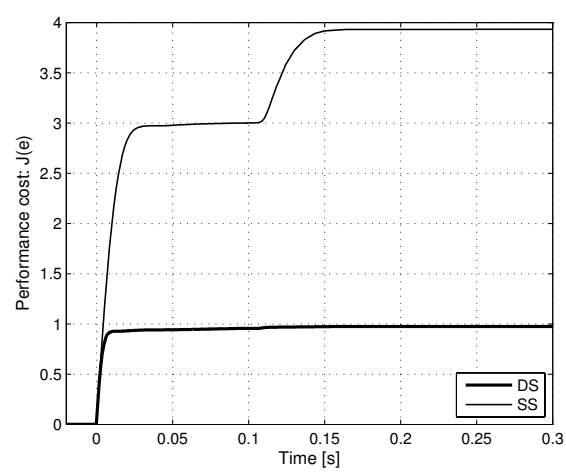

(c) Performance costs

Fig. 9. Experimental tracking control and disturbance rejection.

(10), the simplified controller has lower orders and thus leads to less computational delay. For comparison, we also carry out the experiments for the single-stage (SS) servo system, where the LM is controlled by a PTOS controller [9] and the PA is switched off. The controller is implemented by a real-time DSP system (dSPACE-DS1103) with the sampling frequency of $5 \mathrm{kHz}$. The step reference command and the shock disturbance acting on the LM are, respectively, injected at time 0 and 0.1 second.

From Fig. 9(a), we can see that the PA is effective to speed up the step response and to cancel out the LM position deviation due to the shock disturbance. As such, the dualstage servo significantly outperforms the single-stage servo as shown in Fig. 9(b) in terms of the settling time and disturbance rejection. Further, Fig. 9(c) indicates a much smaller performance cost achieved by the DSA compared to the SS servo. Although the $J_{\text {opt }}$ derived in (14) for the DSA under study can be close to 0 , it is impractical due to unconsidering the actuator saturation limitation, and is thus used for benchmark only.

\section{CONCLUSION}

We have revealed that the tracking and disturbance rejection problems can be decoupled into two independent optimization problems under the 2DOF control framework. Then, this result is applied to the design of the $2 \mathrm{DOF}$ controller for an actual DSA system for disturbance rejection and step tracking in the PA range. Experimental results demonstrated that the proposed DSA control system can significantly speed up the step response and enhance the shock disturbance rejection compared with the single-stage servo system.

\section{REFERENCES}

[1] K. Mori, T. Munemoto, H. Otsuki, Y. Yamaguchi, and K. Akagi, "A dual-stage magnetic disk drive actuator using a piezoelectric device for a high track density," IEEE Trans. Magn., vol. 27, no. 6, pp. 52985300, Nov. 1991.

[2] W. Yao, and M. Tomizuka, "Robust controller design for a dual-stage positioning system," in Proc. Int. Conf. Industrial Electronics, Control, and Instrumentation, 1993, pp. 62-66.
[3] S. Schroeck, W. Messner, and R. McNab, "On compensator design for linear time-invariant dual-input single-output systems," IEEE/ASME Trans. Mechatron., vol. 6, no. 1, pp. 50-57, Mar. 2001.

[4] X. Huang, and R. Horowitz, "Robust controller design of a dual-stage disk drive servo system with an instrumented suspension," IEEE Trans. Magn., vol. 41, no. 8, pp. 2406-2413, Aug. 2005.

[5] G. Herrmann, M. Turner, I. Postlethwaite, and G. Guo, "Practical implementation of a novel anti-windup scheme in a HDD-dual-stage servo system," IEEE/ASME Trans. Mechatron., vol. 9, no. 3, pp. 580592, Sep. 2004.

[6] T. Shen, and M. Fu, "High precision and feedback control design for dual-actuator systems," in Proc. IEEE Conf. Control Applications, 2005, pp. 956-961.

[7] M. Kobayashi, and R. Horowitz, "Track seek control for hard disk dual-stage servo systems," IEEE Trans. Magn., vol. 37, no. 2, pp. 949-954, Mar. 2001.

[8] B. Hredzak, G. Herrmann, and G. Guo, "A proximate-time-optimal control design and its application to a hard disk drive dual-stage actuator system," IEEE Trans. Magn., vol. 42, no. 6, pp. 1708-1715, Jun. 2006.

[9] J. Zheng and M. Fu, "Nonlinear feedback control of a dual-stage actuator system for reduced settling time," IEEE Trans. Contr. Syst. Technol., vol. 16, no. 4, pp. 717-725, Jul. 2008.

[10] M. Vidyasagar, Control System Synthesis: A Factorization Approach, Cambridge, MA: MIT Press, 1995

[11] C. Nett, C. Jacobson, and M. Balas, "A connection between state-space and doubly coprime fractional representations," IEEE Trans. Automat. Contr., vol. AC-29, no. 9, pp. 831-832, Sep. 1984.

[12] W. Su, L. Qiu, and I. Petersen, "Tracking performance limitations under disturbance or uncertainty." in Proc. of IFAC World Congress, 2005.

[13] J. Chen, L. Qiu, and O. Toker, "Limitations on maximal tracking accuracy," IEEE Trans. Automat. Contr., vol. 45, no. 2, pp. 326-331, Feb. 2000.

[14] B. Chang and J. Pearson, "Optimal disturbance reduction in linear multivariable systems," IEEE Trans. Automat. Contr., vol. AC-29, no. 10, pp. 880-887, Oct. 1984.

[15] K. Ohishi, T. Miyazaki, and Y. Nakamura, "High performance ultralow speed servo system based on doubly coprime factorization and instantaneous speed observer," IEEE/ASME Trans. Mechatron., vol. 1, no. 1, pp. 89-98, Mar. 1996.

[16] D. Wang and M. Vidyasagar, "Control of a flexible beam for optimum step response," in Proc. IEEE Int. Conf. Robotics and Automation, 1987, pp. $1567-1572$

[17] I. Shung and M. Vidyasagar, "Control of a flexible robot arm with bounded input: Optimum step responses," in Proc. IEEE Int. Conf. Robotics and Automation, 1987, pp. 916-922.

[18] M. White, M. Tomizuka, and C. Smith, "Rejection of disk drive vibration and shock disturbances with a disturbance observer," in Proc. American Control Conf., 1999, pp. 4127-4131. 